\title{
Outstanding Paper of 2007
}

\author{
Hitoshi KosUGE ${ }^{1}$ and Naoto OHMURA ${ }^{2}$ \\ ${ }^{1}$ Chairman, Outstanding Paper Awards Subcommittee 2007 of JCEJ \\ Department of Chemical Engineering, \\ Graduate School of Science and Engineering, \\ Tokyo Institute of Technology, \\ 2-12-1, Ookayama, Meguro-ku, Tokyo 152-8552, Japan \\ ${ }^{2}$ Editor-in-Chief of JCEJ in 2007 \\ Department of Chemical Science and Engineering, \\ Kobe University, 1-1, Rokkodai-cho, Nada-ku, Kobe-shi, \\ Hyogo 657-8501, Japan
}

Keywords: JCEJ Paper Award, Outstanding Paper

Outstanding Paper Awards Subcommittee of Journal of Chemical Engineering of Japan has assessed the 187 papers published in volume 40 into 2007, and the editorial board finally selected the four papers for JCEJ Paper Awards of 2007; those are the papers on "Incorporation of Capillary-Like Structures into Dermal Cell Sheets Constructed by Magnetic Force-Based Tissue Engineering," "Water Quality Control for Intensive Shrimp Culture Ponds in Developing Countries Using Ammonia-Nitrogen Uptake by Seaweed," "Numerical Analysis of the Mixing Process of a Heterogeneously Viscous System with High Concentration Slurry Liquids in a Stirred Vessel," and "Production of Fuel Gas through the Hydrothermal Gasification of Wastewater Using Highly Active Carbon-Base Catalyst."

JCEJ Paper Award is given for outstanding contribution to chemical engineering documented in a paper published in Journal of Chemical Engineering of Japan. The selection is made by means of a three-stage process consisting of nomination, first round elimination, and point rating. Outstanding Paper Awards Subcommittee of the journal has assessed the 187 papers published in 2007 , corresponding to volume 40 . The subcommittee selected the candidates of the award and the members of the editorial board confirmed the selection. As a result of this process, the editorial board finally selected the following four papers for the award of 2007.

1. "Incorporation of Capillary-Like Structures into Dermal Cell Sheets Constructed by Magnetic Force-Based Tissue Engineering" by Kosuke Ino, Akira Ito, Hirohito Kumazawa, Hideaki Kagami, Minoru Ueda and Hiroyuki Honda, Nagoya University, the Japan Society for the Promotion of Science, and Kyushu University (Ino et al., 2007)

Citation: This research presents a novel methodology to construct 3D vascularized cell sheets using "magnetite cationic liposomes" (MCLs) and "magnetic

Received on July 29, 2008. Correspondence concerning this article should be addressed to H. Kosuge (E-mail address: kosuge.h.ab@m.titech.ac.jp). force-based tissue engineering" (Mag-TE) that will help to overcome the major obstacle in tissue engineering like neovascularization. The technique could be successfully used for vascular stabilization after tissue transplantation, and also for micro patterning of target cells. It will play an important role in the realm of surgery, being welfare to the mankind. The Mag-TE technique is unique and the basic idea has originality. Therefore, this paper deserves the Outstanding Paper Award.

2. “Water Quality Control for Intensive Shrimp Culture Ponds in Developing Countries Using Ammonia-Nitrogen Uptake by Seaweed" by Ryuichi Egashira and Kosuke Sato, Tokyo Institute of Technology (Egashira and Sato, 2007)

Citation: After evaluating the performance of ammonia-nitrogen uptake by the seaweed, the practical use of seaweed to removal ammonia-nitrogen from the intensive shrimp culture ponds in the developing countries has been investigated. When the amount of seaweed in the pond was specified at two steps according to the growth of shrimp, ammonia-nitrogen could be kept sufficiently low enough to cultivate shrimps in the ponds. This is an attractive and practicable research.

3. "Numerical Analysis of the Mixing Process of a Heterogeneously Viscous System with High Concentration Slurry Liquids in a Stirred Vessel" by 
Meguru Kaminoyama, Kazuhiko Nishi, Ryuta Misumi, Tomoyuki Inoue and Hiroshi Takeda, Yokohama National University and Rflow Co., Ltd. (Kaminoyama et al., 2007)

Citation: The mixing process of high concentration slurry liquids in a stirred vessel was analyzed numerically, where the liquids have a plastic rheological property. The dependency of the viscosity on the strain stress was evaluated at local points in the vessel. The mixing time obtained from the numerical analysis agreed with the experimental one fairly sufficiently. The dimensionless mixing time with power consumption was correlated well with Reynolds number and the initial viscosity ratio and the initial concentration difference between the upper and lower liquids. The analysis is useful for understanding the complicated mixing phenomena of high concentration slurry liquids.

\section{4. "Production of Fuel Gas through the Hydro- thermal Gasification of Wastewater Using Highly Active Carbon-Base Catalyst" by Hiroyuki Nakagawa, Akio Namba, Atul Sharma and Kouichi Miura, Kyoto University and National Institute of Advanced Industrial Science and Tech- nology (Nakagawa et al., 2007)}

Citation: This article describes a gasification process of the organic compounds dissolving in an industrial wastewater under hydrothermal conditions. A novel $\mathrm{Ni} / \mathrm{C}$ catalyst developed by the author's group was used for gasification and the effect of various gasi- fication conditions on the reactivity was investigated experimentally. The mass and energy balance of the gasification process were also calculated. The authors showed the gasification product had a potential of the fuel gas. The proposed process was applicable for the energy production process from the wastewater. These results give useful information to the wastewater treatment technology. Therefore, this paper deserves the Outstanding Paper Award.

Prizes are presented at the 40th Autumn Meeting of the Society of Chemical Engineers, Japan.

Congratulations to all the award winners. We would also like to acknowledge the cooperation of the Editors and the time and effort of the subcommittee members.

\section{Literature Cited}

Ino, K., A. Ito, H. Kumazawa, H. Kagami, M. Ueda and H. Honda; "Incorporation of Capillary-Like Structures into Dermal Cell Sheets Constructed by Magnetic Force-Based Tissue Engineering," J. Chem. Eng. Japan, 40, 51-58 (2007)

Egashira, R. and K. Sato; "Water Quality Control for Intensive Shrimp Culture Ponds in Developing Countries Using Ammonia-Nitrogen Uptake by Seaweed," J. Chem. Eng. Japan, 40, 454-462 (2007)

Kaminoyama, M., K. Nishi, R. Misumi, T. Inoue and H. Takeda; "Numerical Analysis of the Mixing Process of a Heterogeneously Viscous System with High Concentration Slurry Liquids in a Stirred Vessel," J. Chem. Eng. Japan, 40, 645-651 (2007)

Nakagawa, H., A. Namba, A. Sharma and K. Miura; "Production of Fuel Gas through the Hydrothermal Gasification of Wastewater Using Highly Active Carbon-Base Catalyst," J. Chem. Eng. Japan, 40, 1210-1215 (2007) 